科 学 通 报

\title{
氧转移反应在合成金属有机化合物中的应用 $-\operatorname{Re}_{2}\left(\mu-\mathrm{L}^{\prime}\right)(\mathrm{CO})_{6} \mathrm{~L}-\mathrm{L}\left(\mathrm{L}-\mathrm{L}=\mathrm{Ph}_{2} \mathbf{P}-\mathrm{CH}_{2}-\mathrm{CH}_{2}-\mathbf{P P h}_{2}\right.$, $\mathrm{L}^{\prime}=\mathrm{Cl}$ 或 $\left.\mathrm{OCH}_{3}\right), \operatorname{Re}(\mathrm{Cl})(\mathrm{CO})_{3} \mathrm{~L}-\mathrm{L}$ 的合成、表征和结构 *
}

\author{
高忆慈 王玉强 史启祯 \\ (兰州大学化学系, 兰州 730000)
}

\section{关链词氛原子转移反应、 $\left(\mathrm{C}_{2} \mathrm{H}_{3}\right)_{3} \mathrm{NO}$ 、取代的金風崖基化合物}

近期报道了研究氧原子转移反应的第一批定量结果 ${ }^{[1]}$. 这种氧原子转移反应是氧供体中 的一个氧原子转移到配合物的炭碳原子上形成 $\mathrm{CO}_{2}$ 而离开体系 (反应式 (1)) 为驱动力, 使反应 得以在温和条件下实现.

$$
\mathrm{LmM}(\mathrm{CO})_{n}+\mathrm{L}^{\prime} \frac{\mathrm{XO}}{-\mathrm{CO}_{2}} \operatorname{LmM}(\mathrm{CO})_{n-1} \mathrm{~L}^{\prime}
$$

本文利用氧原子供体 $\left(\mathrm{C}_{2} \mathrm{H}_{5}\right){ }_{3} \mathrm{NO}$ 的作用, 常温常压下使 $\mathrm{Re}_{2}(\mathrm{CO})_{10}$ 与外来配体 dppe $\left(\mathrm{Ph}_{2} \mathrm{P}-(\mathrm{C}\right.$ $\mathrm{H}_{2}{ }_{2}-\mathrm{PPh}_{2}$ 的缩写以一定的化学计量在溶剂中反应, 成功地合成了三种新型的、含双膦配体的 取代宸基配合物: $\operatorname{Re}_{2}(\mu-\mathrm{Cl})_{2}(\mathrm{CO})_{6} \mathrm{dppe}(\mathrm{I}), \operatorname{Re}_{2}\left(\mu-\mathrm{OCH}_{3}\right)_{2}(\mathrm{CO})_{6} \mathrm{dppe}(\mathrm{II}), \operatorname{Re}(\mathrm{Cl})(\mathrm{CO})_{3}$ dppe (III), 并描述了它们的光谱表征和结构特点. $\mathrm{Kang}^{[2]}$ 预期的这些信息迄今未见报道.

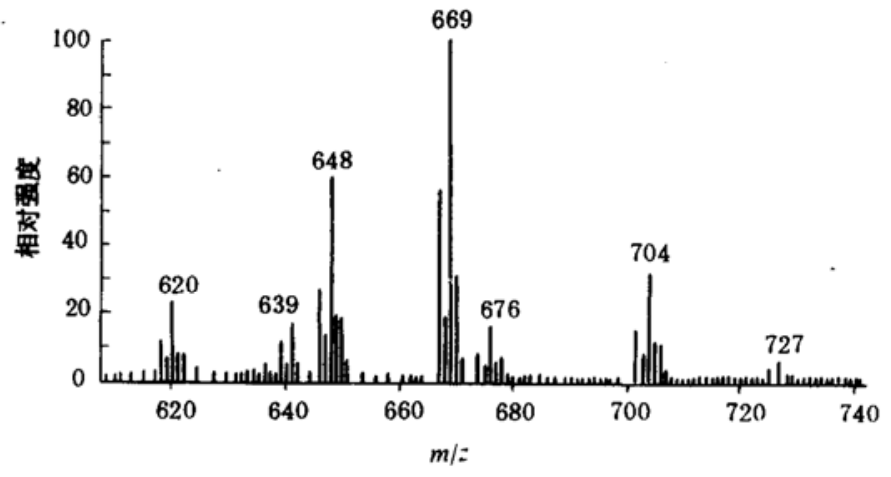

图 $1 \mathrm{ReCl}(\mathrm{CO})_{3} \mathrm{dppe}$ 的质谱图

\section{1 实 验}

\section{1 试剂}

$\mathrm{Re}_{2}(\mathrm{CO})_{10}$ 和 dppe 分别购自美 国 Aldrich 和 Strem 公司. $\left(\mathrm{C}_{2} \mathrm{H}_{5}\right)_{3}$ NO 按文献[3] 提供的方法制备并 提 纯. $\mathrm{CHCl}_{3}, \mathrm{THF}, \mathrm{CH}_{3} \mathrm{OH}$ 和 $\mathrm{CH}_{3} \mathrm{CN}$ 按常规方法干燥并在使用 前蒸出.

\section{2 仪器}

涉及金属宸基化合物的实验 均使用 Schlenk 操作. 元素分析在意大利 1106 型元素分析仪上完成. 反应过程用 170-SX 型 IR 光谱仪跟踪. 固体配合物用 KBr 压片制样在 Nicolet-5DXFT-IR 光谱仪上测 IR 谱图, ${ }^{13} \mathrm{CNMR},{ }^{31} \mathrm{P}$ NMR 和MS(FAB) (图 1) 分别在 FT-80A, Brucker AM-400 核磁共振仪和 VG, 
2AB-MS 质谱仪上测得. 晶体结构用 $\mathrm{R} 3 \mathrm{M} / \mathrm{E} \mathrm{X}$ 射线四圆单晶衍射仪测定.

\section{3 配合物的合成}

(1) $\operatorname{Re}_{2}(\mu-\mathrm{Cl})_{2}(\mathrm{CO})_{6} \mathrm{dppe}$ 和 $\mathrm{Re}(\mathrm{Cl})(\mathrm{CO})_{3} \mathrm{dppe}$ 的合成方法. 在 $\mathrm{Re}_{2}(\mathrm{CO})_{10}(0.3268 \mathrm{~g}, 0.50 \mathrm{mmol})$ 和 $\left(\mathrm{C}_{2} \mathrm{H}_{5}\right)_{3} \mathrm{NO} \cdot \frac{1}{2} \mathrm{H}_{2} \mathrm{O}(0.1232 \mathrm{~g}, 1.52 \mathrm{mmol})$ 的 $\mathrm{CHCl}_{3}(15 \mathrm{~mL})$ 溶液中, 边摚拌边加人 dppe $(0.199 \mathrm{~g}$, $0.50 \mathrm{mmol})$, 室温下持续搅拌 $2 \mathrm{~h}$, 溶液由无色逐渐变为橙黄, 反应液用红外光谱跟踪检测, 直到 $\mathrm{Re}_{2}(\mathrm{CO})_{10}$ 在 $2013 \mathrm{~cm}^{-1}$ 和 $1968 \mathrm{~cm}^{-1}$ 的强吸收峰完全消失. 然后减压蒸熘除去过量溶剂, 浓缩液 转移到硅胶柱色谱上分离, 用 $\mathrm{CHCl}_{3}$ 淋洗, 分段收集组分, 纯化结晶后得到 ( I ) 和 (III), 产率达 $48.5 \%$. C、 $\mathrm{H}$ 的测得值 (\%) 与预期组成的计算值 (\%) 吻合 (见表 1). ( I ) 的单晶是在室温下 从氯仿溶液中重结晶制得.

表 1 配合物元素分析和光谱数据

\begin{tabular}{|c|c|c|c|c|c|}
\hline 配合物 & C, H (\%) & $\begin{array}{l}\text { IR } \\
v_{\mathrm{\infty}}, \mathrm{cm}^{-1 \mathrm{a})}\end{array}$ & $\begin{array}{c}{ }^{13} \mathrm{C} \text { NMR } \\
\delta^{(b)}\end{array}$ & $\begin{array}{ll}{ }^{31} \mathrm{P} & \mathrm{NMR} \\
& \delta^{\text {b) }}\end{array}$ & $\begin{array}{l}\mathrm{M}^{+} \\
m / e\end{array}$ \\
\hline \multirow[t]{7}{*}{ ( I ) } & $37.87 \quad 3.26$ & $2050(\mathrm{~s}), 2034(\mathrm{~s})$ & $190.0(d, 1 c)$ & $7.4(s, 2 p)$ & 1010 \\
\hline & $(38.07)(3.40)$ & 2022 (sh), 1970 (s) & $187.6(\mathrm{~s}, \mathrm{lc})$ & & \\
\hline & & 1942(s), 1915(s) & $186.9(\mathrm{~s}, 1 \mathrm{c})$ & & \\
\hline & & & $128.8-133.4$ & & \\
\hline & & & $(\mathrm{m}, 24 \mathrm{c})$ & & \\
\hline & & & $29.4(s, 2 c)$ & & \\
\hline & & & $J_{p c}=65.8 \mathrm{~Hz}$ & & \\
\hline \multirow[t]{7}{*}{ ( II ) } & & $2021(\mathrm{~s}), 2005(\mathrm{~s})$ & $195.6(\mathrm{~s}, 4 \mathrm{c})$ & $15.2(s, 2 p)$ & 1000 \\
\hline & & $1915(\mathrm{~m}), 1887(\mathrm{~s})$ & $194.3(s, 1 c)$ & & \\
\hline & & 1876(sh) & $193.4(\mathrm{~s}, 1 \mathrm{c})$ & & \\
\hline & & & $128.9-133.8$ & & \\
\hline & & & $(\mathrm{m}, 24 \mathrm{c})$ & & \\
\hline & & & $73.55\left(s, 2^{*} c\right)$ & & \\
\hline & & & $\left({ }^{*} \mathrm{OCH}_{3}\right)$ & & \\
\hline \multirow[t]{7}{*}{ ( III ) } & $49.70 \quad 3.41$ & $2020(s), 2005(s)$ & $192.3(d, 1 c)$ & $31.9(s, 2 p)$ & 704 \\
\hline & $(49.47)(3.44)$ & 1889 (s,sh) & 191.7(d, lc) & & \\
\hline & & & $189.1(\mathrm{~s}, \mathrm{lc})$ & & \\
\hline & & & $128.4-133.4$ & & \\
\hline & & & $(\mathrm{m}, 24 \mathrm{c})$ & & \\
\hline & & & $26.5(\mathrm{~m}, 2 \mathrm{c})$ & & \\
\hline & & & $J_{\mathrm{pc}}=70.6 \mathrm{~Hz}$ & & \\
\hline
\end{tabular}

a) $\mathrm{CHCl}_{3}$ (溶剂), b) $\mathrm{CDCl}_{3}$ (溶剂), ( ) 内为计算值. 
(2) $\operatorname{Re}_{2}\left(\mu-\mathrm{OCH}_{3}\right)_{2}(\mathrm{CO})_{6} \mathrm{dppe}$ 的合成方法. 用类似合成 (I) 和 (III) 的方法使 $\operatorname{Re}_{2}(\mathrm{CO})_{10}$ $(0.3268 \mathrm{~g}, 0.50 \mathrm{mmol})$ 和 $\left(\mathrm{C}_{2} \mathrm{H}_{5}\right)_{3} \mathrm{NO}(0.1232 \mathrm{~g}, 1.52 \mathrm{mmol}$ 溶于 THF-MeOH $(5: 1, V / V)$ 的混合溶剂 中, 后续步骤同反应式 (1), 得到配合物 (II), 产率约 $40 \%$.

\section{2 结 果 和 讨 论}

(I) 和 (III) 直接用 $\mathrm{Re}_{2}(\mathrm{CO})_{10}$ 和 dppe 反应在氧原子供体作用下一步取得产物:

$$
\begin{aligned}
\operatorname{Re}_{2}(\mathrm{CO})_{10}+\text { dppe } \frac{3\left(\mathrm{C}_{2} \mathrm{H}_{5}\right)_{3} \mathrm{NO}, \mathrm{N}_{2}}{\mathrm{CHCl}_{3}, 2 \mathrm{~h}} & \operatorname{Re}_{2}(\mu-\mathrm{Cl})_{2}(\mathrm{I})(\mathrm{CO})_{6} \text { dppe (I ) } \\
& +\operatorname{Re}(\mathrm{Cl})(\mathrm{III})(\mathrm{CO})_{3} \mathrm{dppe}(\mathrm{II})
\end{aligned}
$$

反应式 (2) 形成的产物是特别有趣的,分子中的 $\mathrm{Cl}$ 配体来自 $\mathrm{CHCl}_{3}$ 溶剂. 这一假设在反应式 (3) 的产物中得到了证实. 在 THF- $\mathrm{CH}_{3} \mathrm{OH}$ 混合溶剂中制得一个由 $-\mathrm{OCH}_{3}$ 桥代替了 $\mathrm{Cl}$ 桥的 结构类似的化合物 (II) .

$$
\mathrm{Re}_{2}(\mathrm{CO})_{10}+\text { dppe } \frac{3\left(\mathrm{C}_{2} \mathrm{H}_{5}\right)_{3} \mathrm{NO}, \mathrm{N}_{2}}{\mathrm{THF}-\mathrm{CH}_{3} \mathrm{OH}, 2 \mathrm{~h}} \operatorname{Re}_{2}(\mu-\mathrm{OMe})_{2}(\mathrm{CO})_{6} \mathrm{dppe} \text { (II) }
$$

配合物的元素分析和光谱数据及其归属列于表 1. 归纳所有谱学数据, 可以确认分子 (I) 和 (II) 中的 L-L 配体桥连在一起的两个 $\operatorname{Re}$ 原子 (每个 $\operatorname{Re}$ 分别与一个 $\mathrm{P}$ 键合) 占据的是平 伏键 的位置, (I) 的晶体结构图 (图 2)也支持了这一结论. (II) 的 IR 谱图上除了 $v_{\infty}$ 峰的特征吸收

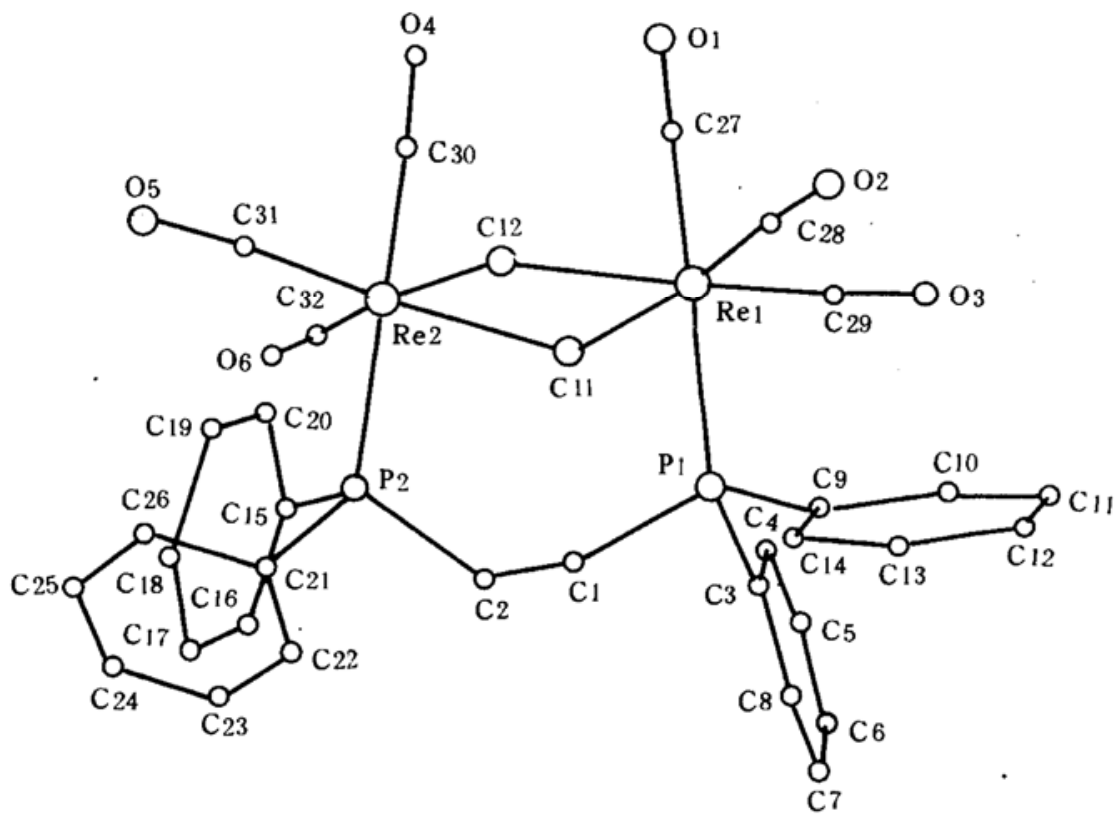

图 $2 \mathrm{Re}_{2}(\mu-\mathrm{Cl})_{2}(\mathrm{CO})_{6} \mathrm{dppe}$ 的非氢分子结构图

外,还提供了分子中有 $\mathrm{OCH}_{3}$ 基存在的信息: 它的对称 $v_{\mathrm{C}-\mathrm{H}}$ 位于 $2830 \mathrm{~cm}^{-1}$ (一般碳甲基在 2872 $\pm 10 \mathrm{~cm}^{-1}$ ); 对称的 $\delta_{\mathrm{C}-\mathrm{H}}$ 位于 $1445 \mathrm{~cm}^{-1}$ (一般碳甲基在 $1375 \mathrm{~cm}^{-1}$ ). (III) 的 IR 谱图上的 $v_{\infty}$ 谱带 特征 (即红外活性吸收峰的强度和数目: $\mathrm{s}, \mathrm{s}, \mathrm{s}(\mathrm{sh})$ ) 为这个 (L-L) $\mathrm{L}^{\prime} \mathrm{M}(\mathrm{CO})_{3}$ 型取代基化合物具 有面式八面体结构提供了既简单又方便的信息 ${ }^{[4]}$. 
配合物 (I) 的分子结构(图 2)表明, 它是由氯桥和 dppe 桥连在一起的两个 $\operatorname{Re}(\mathrm{CO})_{3}$ 单体组 成. 对每一个 $\operatorname{Re}$ 原子而言, 配位数为 6 , 是一个畸变的八面体几何构型, 两个 $\operatorname{Re}$ 的八面体共 享一个由桥氯原子组成的共用边. 中心的非氢原子 $(\mathrm{Re})_{2}(\mathrm{CO})_{4} \mathrm{Cl}_{2}$ 不在同一平面上, $\mathrm{Cl}_{2} \mathrm{Cl}_{1} \mathrm{Re}_{1} \mathrm{C}_{28} \mathrm{C}_{28}$ 和 $\mathrm{Cl}_{2} \mathrm{Cl}_{1} \mathrm{Re}_{2} \mathrm{C}_{31} \mathrm{C}_{32}$ 之间的两面角为 14.1. $\mathrm{Re}_{2}$ 和 $\mathrm{Re}_{1}$ 之间的非键距离为 $3.681 \AA$ 远远大于 $\operatorname{Re}_{2}(C O)_{10}$ 中的 $\operatorname{Re}-\operatorname{Re}$ 键长 $(3.041 \AA)^{[9}$, 这与 Wood ${ }^{[2]}$ 和我们自己预期的不同. $\mathrm{Re}-\mathrm{CO}$ 键的平均键长为 $1.897 \AA$ 比 $\mathrm{Re}_{2}(\mathrm{CO})_{10}$ 中的相应键长 $(1.975 \AA)^{16}$ 缩短 $0.078 \AA$, 显然这 是由于 dppe 的配位推动了密集在中心金属上的电子云向踦碳反馈促使 $\mathrm{Re}-\mathrm{CO}$ 键级增大的 反映. 与 $\mathrm{P}$ 反位的崖碳基团的 $\mathrm{Re}-\mathrm{CO}$ 键 (平均键长为 $1.924 \AA$ ) 和 $\mathrm{C}-\mathrm{P}$ 键 (平均键长为 1 . $1.166 \AA$ ) , 与反位没有任何 $\pi$-特性的配体的类似物种的对应键长比均有明显的拉长和缩短, 可能与配合物中处在对位的 $\mathrm{CO}$ 和膦两种 $\pi$-酸配体相互竞争同一金属 $d$ 轨道的 $d \pi$ 电子密度 有关. $\mathrm{M}-\mathrm{CO}$ 和 $\mathrm{C}-\mathrm{O}$ 键长之间这种此长彼消的现象在过渡金属羭基配合物中是一种普遍的 规律.

\section{参考 文 献}

[1] a. Shi, Y. L., Gao, Y. C., Shi, Q. Z. et al., Organometallic, 1987, 6: 1528.

b. Gao, Y. C., Shi, Q. Z., Kershner, D. L. et al., Inorg. Chem., 1988, 27: 188.

c. Shen, J. K., Shi, Y. L., Gao, Y. C. et al., J. Am. Chem. Soc., 1988, 110; 2424.

d. Basolo, F., Polyhedron, 1990, 9:1503.

[2] Kang, W. L., William, T., Organometallic, 1984, 3:404.

[3] Cunningham, D. W., Workman, M. O., J. Inorg. Nucl. Chem., 1971, 3861.

[4] Charles, M. L., Fundamental Transition Metal Organometallic Chemistry, Books/Cole Publishing Company, Monterrey, California, 1985, 13-83.

[5] Andy Hor, T. S., J. Organomet. Chem., 1987, 319: 213.

[ 6] Churchill, M. R., Amoh, K. N., Inorg. Chem., 1981, 20: 1609. 